\title{
The Lateral Eddy Viscosity Derived from the Decay of Oceanic Mesoscale Eddies
}

\author{
Qiuyang Li ${ }^{1}$, Liang Sun ${ }^{*}$, Chi Xu${ }^{2}$ \\ ${ }^{1}$ School of Earth and Space Sciences, University of Science and Technology of China, Hefei, China \\ ${ }^{2}$ State Key Laboratory of Tropical Oceanography, South China Sea Institute of Oceanology, Chinese Academy of Sciences, \\ Guangzhou, China \\ Email: *sunl@ustc.edu.cn, sunl@ustc.edu
}

How to cite this paper: Li, Q.Y., Sun, L. and Xu, C. (2018) The Lateral Eddy Viscosity Derived from the Decay of Oceanic Mesoscale Eddies. Open Journal of Marine Science, 8, 152-172. https://doi.org/10.4236/ojms.2018.81008

Received: December 6, 2017

Accepted: January 28, 2018

Published: January 31, 2018

Copyright ( $) 2018$ by authors and Scientific Research Publishing Inc. This work is licensed under the Creative Commons Attribution International License (CC BY 4.0).

http://creativecommons.org/licenses/by/4.0/

(c) (i) Open Access

\begin{abstract}
The relationship of lateral eddy viscosity depending on length scale is estimated with the decay rate of mesoscale eddies identified from sea level anomaly of satellite observations. The eddy viscosity is expressed in terms of the mesoscale eddy parameters according to vortex dynamics. The census of mesoscale eddies shows, in general, that the eddy numbers obey the e-folding decay laws in terms of their amplitude, area and lifetime. The intrinsic values in the e-folding laws are used to estimate the lateral eddy viscosity. Dislike the previous theory that diffusivities are proportional to the length square, the eddy mixing rates (diffusivity and viscosity) from satellite mesoscale eddy datasets are proportional to $r_{s}$ to power of 1.8 (slightly less than 2), where $r_{s}$ is the radius of eddy with radius larger than the Batchelor scale. Additionally, the extrapolation of the eddy mixing to the molecule scale implies that the above power laws may hold until the value of $r_{s}$ is less than $\mathrm{O}(1 \mathrm{~m})$. These mixing rates with the new parameterizations are suggested to use in numerical schemes. Finally, the climatological distributions of eddy viscosity are calculated.
\end{abstract}

\section{Keywords}

Eddy Mixing, Eddy Viscosity, Mesoscale Eddy

\section{Introduction}

In numerical ocean models, effects of the mesoscale eddies should be accurately represented or parameterized to study the general ocean circulations, since satellite observations [1] [2] indicated that oceanic mesoscale eddies (spatial scales on the order of $10-100 \mathrm{~km}$ ) are an important component of the oceanic circulation 
[3] [4]; in particular, eddies play critical role for the major current systems, such as the Antarctic Circumpolar Current (ACC) [5]. As numerical models have horizontally resolution vary over a wide range, from "coarse resolution" ( $\sim 1^{\circ}$ grid), "eddy permitting" $\left(<1 / 4^{\circ}\right.$ grid) to "eddy resolving" $\left(<0.1^{\circ}\right.$ grid $)$, as well as the very crude resolution used in the ocean component of climate models [6]. In general, the eddy mixing rates (diffusivity and viscosity) are parameterized with a simple relationship proportional to the length square and independent of the time and space [7]. However, such simple relationship is not fully supported by the previous field observations and recent parameterizations.

For passive tracers from the oceanic observations, the isopycnal eddy diffusivity and viscosity may is on the order of $10^{3} \mathrm{~m}^{2} / \mathrm{s}$ [7], it varies from $10^{2} \mathrm{~m}^{2} / \mathrm{s}$ [8] [9] [10] to more than $10^{4} \mathrm{~m}^{2} / \mathrm{s}$ [11] [12] [13]. The results show that eddy mixing rates depend not on a simple relationship as suggested before. Thus one problem is the appropriate choice of parameterizations for the eddy properties, e.g." "what is the appropriate choice of the parameterizations and numerical schemes?" [6].

The diffusivities used in different approaches, e.g., the along-isopycnal diffusivity for tracers [14], the effective diffusivity [15] and the Osborn-Cox diffusivity [16], are different, although they all look similar [17]. According to the theory, diffusivities are proportional to the length square and independent of the time scale; in fact, the effective diffusivity $k_{e}$ is defined as $k_{e}=k_{m} L_{\text {eff }}^{2} / L_{\min }^{2}$, where $L_{\text {eff }}$ is the equivalent length of a tracer contour that has been stretched by eddy stirring, $L_{\min }$ is the minimum possible length (a.k.a. the Batchelor scale) of such a contour, and $k_{m}$ is the small-scale background diffusivity [18]. The Osborn-Cox diffusivity $k_{O C}$ is similar to $k_{e}$, but $L_{\text {eff }}$ and $L_{\min }$ are calculated from the spatial gradient of the tracer itself and the tracer's disturbance, respectively [19]. Both lengths $L_{\text {eff }}$ and $L_{\min }$ can be calculated from the Sea Level Anomaly (SLA) data, and the small-scale background diffusivity $k_{m}$ is set according to some field observations.

It is expected that the resulting diffusivities are independent of any unknown parameters [20]. Assume that the spatial resolution is sufficiently small so that it is suitable for explicit small-scale diffusivity, the resulting diffusivities are surprisingly different. The effective diffusivity $k_{e}$ is proportional to $k_{m}^{1 / 4}$ (exponent of 0.24 other than 0.25 used here), given a $k_{m}$ in the range of 10 to $10^{2} \mathrm{~m}^{2} / \mathrm{s}$ [18]. Additionally, $k_{O C}$ depends weakly on $k_{m}: k_{O C} \propto k_{m}^{-1 / 5}$, given $k_{m}$ in the range of $0.5-5 \mathrm{~m}^{2} / \mathrm{s}$ [19]. Both diffusivities depend on $k_{m}$, but $k_{e}$ is directly proportional to $k_{m}$ and $k_{O C}$ is inversely proportional to $k_{m}$.

Thus the simple relationship that diffusivities are proportional to the length square should be modified according to these previous studies. The above inconsistent results reveal the possibility that $k_{m}$ is not proportional to $L_{\min }^{2}$; instead, it is proportional to $L_{\min }^{n}(n<2)$, i.e., $k_{m}=k L_{\min }^{n}$, where $k$ is the molecule-scale viscosity, because the time scale dependency on the length scale for the current range of spatial resolution range, as do $k_{e}$ and $k_{O C}$ to $L_{e f f}$ and other length scales. According to the above relations of $k_{e}$ and $k_{O O}$ it is found that $n=3 / 2$ for 
$k_{m}$ in the range of 10 to $10^{2} \mathrm{~m}^{2} / \mathrm{s}$ and $n=5 / 3$ for $k_{m}$ in the range of 0.5 to $5 \mathrm{~m}^{2} / \mathrm{s}$. When $n=5 / 3$, it is the classic $-5 / 3$ power law of energy dissipation for the wave number in 2-dimensional or quasi-geostrophic flow [21]. Only when the resolution is smaller, can $k_{O C}$ become truly independent of $k_{m}$ [19], where $n=2$ holds.

It is hypothesis that eddy viscosity, similar to eddy diffusivity, is proportional to $L_{\min }^{n}(n<2)$. To test this hypothesis, the accumulated long-term satellite altimetry SLA data are used. Benefiting from studies of eddy statistics [4] [22] [23] [24], we use the fluid dynamic theory to estimate the eddy viscosity from the SLA data. It is known that the eddy viscosity, unlike the eddy diffusivity, can be directly derived from the kinetic energy dissipation rate [21].

The paper is organized as follows. In Section 2, we first describe the eddy identification and tracking datasets used in our study, the relation between eddy viscosity and eddy properties based on vortex theory is established; in addition, the intrinsic eddy parameters are calculated from eddy statistics. In Section 3, we use two examples to present the method for estimating eddy viscosities; eddy viscosities in the South China Sea (SCS), the ACC and the global regions from different datasets are calculated. In Section 4, we discuss the relationships between the average parameters and the intrinsic parameters. In Section 5, we draw the conclusions.

\section{Data and Methods}

\subsection{Data}

The SLA data used here are the merged and gridded satellite product of MSLA (Maps of Sea Level Anomaly) produced and distributed by AVISO

(http://www.aviso.oceanobs.com/) based on TOPEX/Poseidon, Jason 1, ERS - 1, and ERS-2 data [2]. The data were corrected for all geophysical errors, and the currently available version has a $0.25^{\circ} \times 0.25^{\circ}$ resolution of the global ocean. Three datasets will be used in this study.

The first eddy dataset is based on the weekly SLA fields in Version 3 of the AVISO data taken from http://cioss.coas.oregonstate.edu/eddies/, which is based on the methods by Chelton et al. (2011) [4]. In their eddy identification method includes three criteria: the amplitude, area and lifetime of the eddy must be larger than $1 \mathrm{~cm}, 8$ pixels and 4 weeks. This 19.5-year (1992-2011) version retains only those eddies with lifetimes of 4 weeks or longer, and the trajectories are available at 7-day time steps. There are a total of 215,184 eddies in this dataset. We denote this dataset as the Chelton dataset.

The second dataset is based on the 20-year (1993-2012) daily SLA fields of the AVISO data. The ocean eddies were identified by the SLA extremes and a sufficient number of neighboring regions, these criteria are similar to those of the previous method [4], except that the eddies were subjected to a mononuclear eddy constraint [22] [23] [24]. Additionally, the amplitude, area and lifetime of the eddies must be larger than $6 \mathrm{~cm}, 17$ pixels and 30 days. Compared with the smaller amplitude cut-off values used in previous studies, the cut-off values used 
in this dataset were as large as the measurement error of the SLA data [25]. Thus, the total number of identified in this dataset, 116,778, is smaller than in the Chelton dataset. We denote this dataset as the Li dataset.

The third eddy dataset is also based on the weekly SLA fields of the AVISO data from 1993 to 2007, where the Okubo-Weiss method [26] [27]) was used to identify eddies in the South China Sea (SCS). Any eddies with a lifetime shorter than 30 days and spatial radius smaller than $45 \mathrm{~km}$ were discarded. There were approximately $32.8 \pm 3.4$ eddies observed by satellite each year from 1993 to 2007. The radius of these eddies ranges from approximately $46.5 \mathrm{~km}$ to 223.5 $\mathrm{km}$, with a mean value of approximately $87.4 \mathrm{~km} \mathrm{[27].} \mathrm{We} \mathrm{denote} \mathrm{this} \mathrm{dataset} \mathrm{as}$ the Xiu dataset.

\subsection{Eddy Viscosity}

In this study, we directly calculate the eddy viscosity using formula from vortex dynamics. For an incompressible flow with density $\rho$ and velocity $\boldsymbol{u}$, the balance of eddy kinetic energy (EKE) $e_{k}=1 / 2 \rho \boldsymbol{u} \cdot \boldsymbol{u}$ is

$$
\frac{\mathrm{d} e_{k}}{\mathrm{~d} t}=\boldsymbol{F} \cdot \boldsymbol{u}-\Phi+B \text {. }
$$

where $F$ is body force per unit mass, $B$ is EKE flux at the boundary, and $\Phi$ is the dissipation rate

$$
\Phi=\rho v_{e} \xi^{2} .
$$

where $v_{e}$ is the eddy viscosity and $\xi=\nabla \times \boldsymbol{u}$ is vorticity. Most eddies in the open ocean are neutrally buoyant; thus, there is no body force and no net EKE flux at the boundary. Hence, the balance of EKE is,

$$
\frac{1}{\rho} \frac{\mathrm{d} e_{k}}{\mathrm{~d} t}=v_{e} \xi^{2} .
$$

To the lowest order approximation, oceanic mesoscale eddies have a universal profile [28], i.e., the Gaussian shape of the Taylor vortex [29]. The sea level height anomaly $h$ along radius $r$ for a Gaussian eddy is as follows:

$$
h=A \exp \left(-\frac{r^{2}}{2 r_{s}^{2}}\right) \text {. }
$$

where $A$ and $r_{s}$ are the amplitude and radius of maximum speed, respectively [4] [29] [30]). The velocity of an eddy in geostrophy is

$$
V_{\theta}=\frac{g A r}{f 2 r_{s}^{2}} \exp \left(-\frac{r^{2}}{2 r_{s}^{2}}\right) \text {. }
$$

where $g$ and $f$ are the gravity acceleration and Coriolis parameter. Thus, the total EKE of the eddy is

$$
E=\int_{0}^{2 \pi} \int_{0}^{\infty} \frac{1}{2} V_{\theta}^{2} r \mathrm{~d} r \mathrm{~d} \theta=\frac{\pi}{2}\left(\frac{g A}{f}\right)^{2} .
$$

The vorticity of the eddy in the geostrophic approximation is 


$$
\xi=\frac{1}{r} \frac{\mathrm{d}}{\mathrm{d} r}\left(V_{\theta} r\right)=\frac{2 g A}{f r_{s}^{2}}\left(1-\frac{r^{2}}{2 r_{s}^{2}}\right) \exp \left(-\frac{r^{2}}{2 r_{s}^{2}}\right) .
$$

The enstrophy of the eddy is

$$
Q=\int_{0}^{2 \pi} \int_{0}^{\infty} \frac{1}{2} \xi^{2} r \mathrm{~d} r \mathrm{~d} \theta=\pi\left(\frac{g A}{f r_{s}}\right)^{2} .
$$

According to Equation (3), the EKE dissipation rate directly linked to the enstrophy [31]

$$
\frac{\mathrm{d} E}{\mathrm{~d} t}=-2 v_{e} Q
$$

For non-rotating fluid, the available gravitational potential energy (AGPE) of a vortex is small; it plays no important role in eddy dynamics, and thus often being ignored. However, AGPE in rotating stratified fluid plays quite important role. In fact, AGPE of the oceanic mesoscale eddy is larger than the EKE in general; on average, the AGPE is approximately 1.7 times the EKE [32]. Because of the continuous conversion between EKE and AGPE, dissipation of the EKE and AGPE are closely linked to each other during the decay of eddies; thus, the eddy viscosity $V_{e}$ introduced in Equation (2) should be multiplied by a factor of $C$ (ratio of total mechanical energy to EKE), and $C \sim 1+1.7=2.7$, as discussed above. For the Gaussian-shaped eddy, this leads to

$$
v_{e}=-\frac{C}{2 Q} \frac{\mathrm{d} E}{\mathrm{~d} t}=-\frac{C r_{s}^{2}}{4 A} \frac{\mathrm{d} A}{\mathrm{~d} t}=-\frac{C L a}{4 \pi} .
$$

where

$$
\begin{gathered}
L=\pi r_{s}^{2} / A=S / A . \\
a=\mathrm{d} A / \mathrm{d} t .
\end{gathered}
$$

$L$, $a$ and $S$ are the length parameter, the amplitude decaying rate and the horizontal area of a circle with radius $r_{s}$. Thus, using observations and Equation (11), we can estimate the horizontal eddy viscosity.

There are several length scales in our analysis: the radius of maximum speed $r_{s}$, the e-folding decay radius $r_{e}$, and the effective radius $r_{\text {eff }}$ The effective radius $r_{\text {eff }}$ is defined to be the radius of the circle that has the same area as the region within the eddy perimeter [4]. The regression relationships, $r_{s}=0.44 r_{\text {eff }}$ and $r_{s}=$ $0.707 r_{e}$, are useful in comparing the results from different datasets [4], note that the eddy area is expressed as effective radius $r_{\text {eff }}$ in original datasets [4] [22] [27]). Both $A$ and $S$ can be obtained from the census statistics of mesoscale eddies [4] [27] [33]. In contrast, the amplitude decaying rate a was discussed in a few studies only [27] [33].

Note that lateral eddy viscosity discussed above is defined for the evolution of an eddy; thus, it may be interpreted as what an observer moving with the eddy can see. In this sense, this analysis can apply to the data collected by tracing the time evolution of individual eddies from satellite altimetry data. Thus, it is a parameter 
defined in the Lagrangian coordinates, so that it can be used as the viscosities for the whole domain in the ocean; however, whether such parameter can be directly used to the commonly used oceanic numerical models remains unclear because such models are defined in Eulerian coordinates.

\subsection{Intrinsic Parameters}

As mentioned above, we need to calculate the decaying rate a of eddy amplitude. The simplest method is to estimate a from the time evolution of eddy amplitude. A simple example is shown in Figure 1, where the blue and red lines indicate the time regressions of eddy amplitude. However, fitting the decay rate of eddies one by one is rather cumbersome, such an approach cannot be applied to a large dataset. Alternatively, we can calculate a from the time evolution of eddy amplitude, i.e., we will use eddy track data to calculate the regression,

$$
A=a T+b .
$$

where $T$ is the lifetime of the eddy. However, this regression is quite noisy, as shown in Figure 8 of Xiu et al. (2010) [27]. Thus, we calculate a from the number-amplitude and number-lifetime relations (e.g. Figure 1).

The amplitude decay rate can be calculated from the eddy number distributions. In general, there are two different types of statistics in the literature (e.g., [4]). One is the number of eddies with lifetimes of $N_{t}(T)$; the other is the number of eddies with amplitudes of $N_{a}(A)$. If the amplitude-lifetime relation is linear, such as Equation (11), then

$$
N_{a}(A)=N_{a}(a T+b)=N_{t}(T) .
$$

On the other hand, if we have statistics of both $N_{t}(T)$ and $N_{a}(A)$, we can use Equation (13) to derive the amplitude-lifetime relation. Specifically, the number of eddies in the global oceans may obey the following e-folding decay laws in terms of its amplitude, area and lifetime

$$
\begin{aligned}
& N_{a}(A)=N_{a 0} \mathrm{e}^{-\mathrm{A} / A_{i}} . \\
& N_{s}(S)=N_{s 0} \mathrm{e}^{-S / S_{i}} . \\
& N_{t}(T)=N_{t 0} \mathrm{e}^{-T / T_{i}} .
\end{aligned}
$$

where $A_{i}, S_{i}$ and $T_{i}$ are the intrinsic eddy amplitude, area and lifetime, respectively, and $N_{a s} N_{s o}$ and $N_{t o}$ are the eddy numbers when $A=S=T=0$. Then, substituting Equation (12) into Equation (14a), and using Equation (13), it yields to $N_{a}(A)=N_{a 0} \mathrm{e}^{-\mathrm{A} / A_{i}}=N_{a 0} \mathrm{e}^{-(a T+b) / A_{i}}=N_{t 0} \mathrm{e}^{-T / T_{i}}$. Therefore, we can use Equation (13) to evaluate $v_{e}$ with the intrinsic parameters.

$$
\begin{aligned}
a & =A_{i} / T_{i} . \\
L & =S_{i} / A_{i} . \\
v_{e} & =\frac{C}{4 \pi} \frac{S_{i}}{T_{i}} .
\end{aligned}
$$






(a)

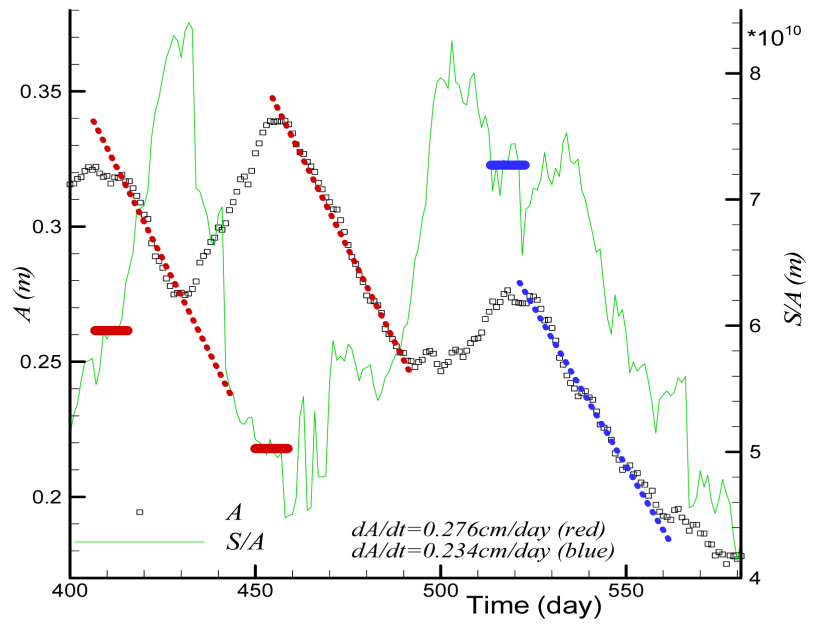

(b)

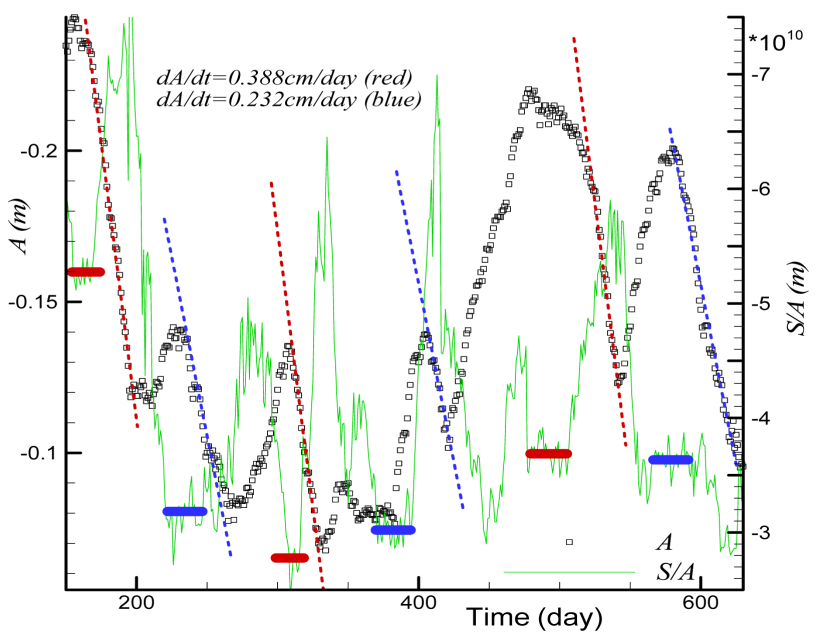

(c)

Figure 1. (a) Two long-term eddy tracks in the ocean. (b) Time series of the amplitude and the ratio of the area to the amplitude for anti-cyclonic eddy 1 . The dashed red and blue lines indicate the linear decay periods, and the amplitude decaying rates are regressed from the lines. Meanwhile, the ratio of S/A is also marked by heavy horizontal red and blue bars; (c) The same as (b) but for cyclonic eddy 2 . 
We will use this method to calculate the eddy viscosity in this study. The intrinsic parameters are obtained from linear regression of e-folding decays (Figures 2-5). For Chelton dataset, the present linear regression of 16 weeks (Figure 5(c)) agrees well with the optimal value of 16 weeks obtained from a stochastic model with the same dataset [34].

\section{Results}

\subsection{Two Examples}

There are two long-lived eddies detected by automated eddy identification and tracking algorithm in the Li dataset (Figure 1(a)). The first anticyclonic eddy moved from $\left(111^{\circ} \mathrm{E}, 36^{\circ} \mathrm{S}\right)$ to $\left(105^{\circ} \mathrm{E}, 32.5^{\circ} \mathrm{S}\right)$ from January 2005 to July 2005. During this time, both the amplitude and the area of the eddy changed substantially. During 3 time intervals the eddy amplitude monotonically decreased (Figure 1(b)). We use a linear regression to fit the decay of eddy's amplitude (red and blue dashed lines) according to Equation (12). The amplitude decaying



(a)



(b)

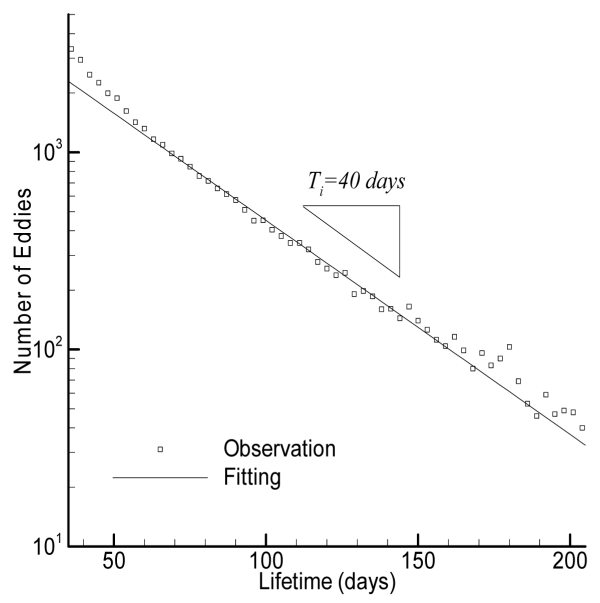

(c)

Figure 2. The power laws for eddy parameties in the ACC based on Li dataset, where the vertical axis is eddy number (in logarithm scale): (a) The number of eddies vs their amplitude; (b) The number of eddies vs their area; (c) The number of eddies vs their lifetime. 


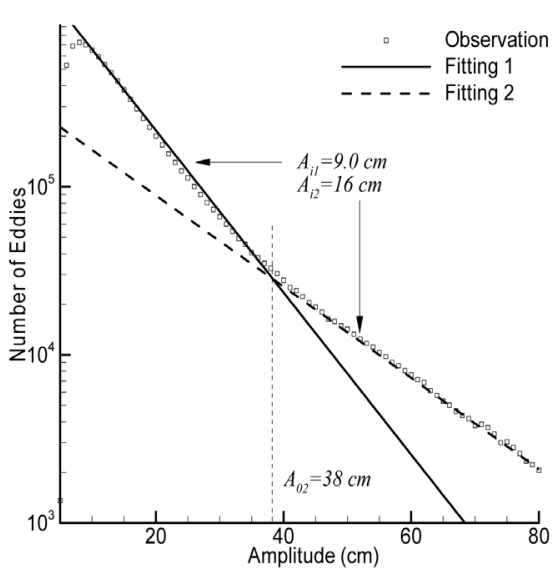

(a)

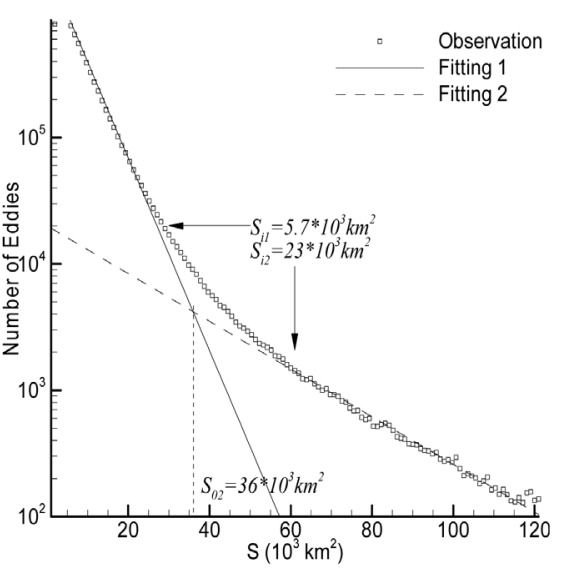

(b)

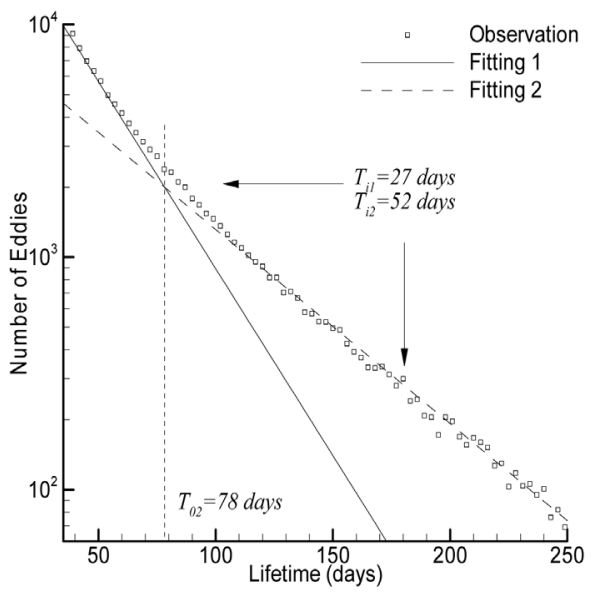

(c)

Figure 3. The same as in Figure 2 but for global oceans. There are critical values $\left(A_{02}=\right.$ $38 \mathrm{~cm}, S_{02}=36 \times 10^{3} \mathrm{~km}^{2}$ and $T_{02}=78$ days) separating the e-folding decay laws into two segments.

rate $a$ is approximately $0.276 \mathrm{~cm} /$ day $\left(3.2 \times 10^{-8} \mathrm{~m} / \mathrm{s}\right)$ for the red lines and 0.234 $\mathrm{cm} /$ day $\left(2.7 \times 10^{-8} \mathrm{~m} / \mathrm{s}\right)$ for the blue line. Meanwhile, the length parameter $(L=$ $S / A)$ is also plotted as the green curve in Figure $1(\mathrm{~b})$. This ratio is roughly the same before each time when eddy amplitude starts to decrease, as marked by the red/blue horizontal lines in Figure 1(b). With the values in Figure 1(b), we can calculate the eddy viscosities in 3 different time periods, as listed in Table 1. The viscosities detected from these episodes are very similar $\left(356-429 \mathrm{~m}^{2} / \mathrm{s}\right)$.

Cyclonic eddy 2 moved from $85^{\circ} \mathrm{E}, 33.5^{\circ} \mathrm{S}$ to $67^{\circ} \mathrm{E}, 36^{\circ} \mathrm{S}$ from November 1995 to December 1997. During this time period, both the amplitude and the area of the eddy changed substantially. There are 6 time intervals when the amplitude monotonically decreased (Figure $1(\mathrm{c})$ ). Similar to eddy 1371833, the amplitude decaying rate a is approximately $0.388 \mathrm{~cm} /$ day $\left(4.5 \times 10^{-8} \mathrm{~m} / \mathrm{s}\right)$ for the red lines and $0.232 \mathrm{~cm} /$ day $\left(2.7 \times 10^{-8} \mathrm{~m} / \mathrm{s}\right)$ for the blue line. The eddy viscosities in these time periods are listed in Table 1. Compared with the values in Figure 1(b), the values $\left(178-521 \mathrm{~m}^{2} / \mathrm{s}\right)$ are much more diverse in this case. Nevertheless, in these 


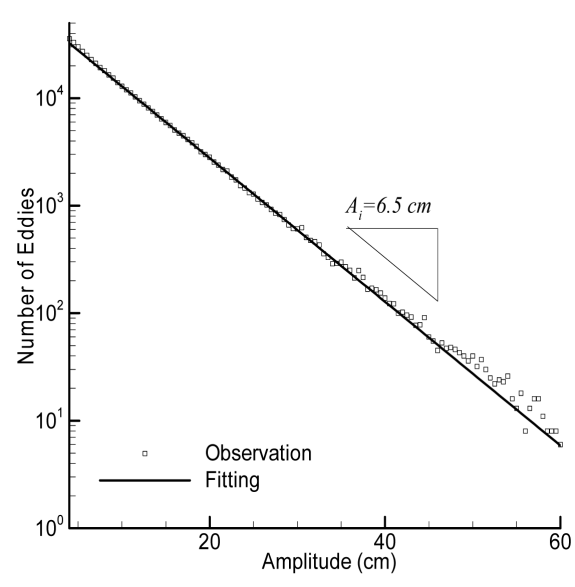

(a)

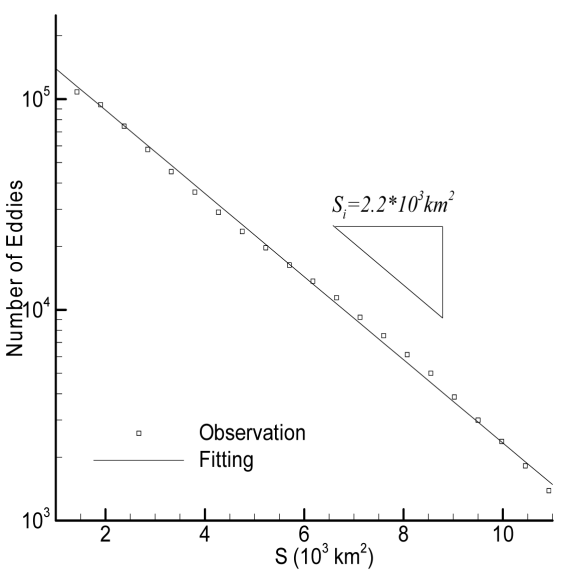

(b)



(c)

Figure 4. The eddy power laws in the ACC based on the Chelton dataset. (a) The number of eddies vs their amplitude. (b) The number of eddies vs their area. (c) The number of eddies vs their lifetime.

Table 1. The isopycnal eddy parameters in different periods for two long-lived eddies

\begin{tabular}{ccccc}
\hline Period & $a(\mathrm{~m} / \mathrm{s})$ & $A(\mathrm{~m})$ & $S / A(\mathrm{~m})$ & $V_{e}\left(\mathrm{~m}^{2} / \mathrm{s}\right)$ \\
\hline A1 & $3.2 \times 10^{-8}$ & 0.32 & $5.9 \times 10^{10}$ & 405 \\
A2 & $3.2 \times 10^{-8}$ & 0.34 & $5.0 \times 10^{10}$ & 356 \\
A3 & $2.7 \times 10^{-8}$ & 0.27 & $7.3 \times 10^{10}$ & 429 \\
B1 & $4.5 \times 10^{-8}$ & 0.30 & $5.2 \times 10^{10}$ & 521 \\
B2 & $2.7 \times 10^{-8}$ & 0.14 & $3.2 \times 10^{10}$ & 189 \\
B3 & $4.5 \times 10^{-8}$ & 0.135 & $2.7 \times 10^{10}$ & 275 \\
B4 & $2.7 \times 10^{-8}$ & 0.14 & $3.0 \times 10^{10}$ & 178 \\
B5 & $4.5 \times 10^{-8}$ & 0.21 & $3.6 \times 10^{10}$ & 356 \\
B6 & $2.7 \times 10^{-8}$ & 0.20 & $3.6 \times 10^{10}$ & 213 \\
\hline
\end{tabular}

examples the eddy viscosities inferred from observations are relatively small, on the order of $\left(\sim 300 \mathrm{~m}^{2} / \mathrm{s}\right)$. 




(a)



(b)

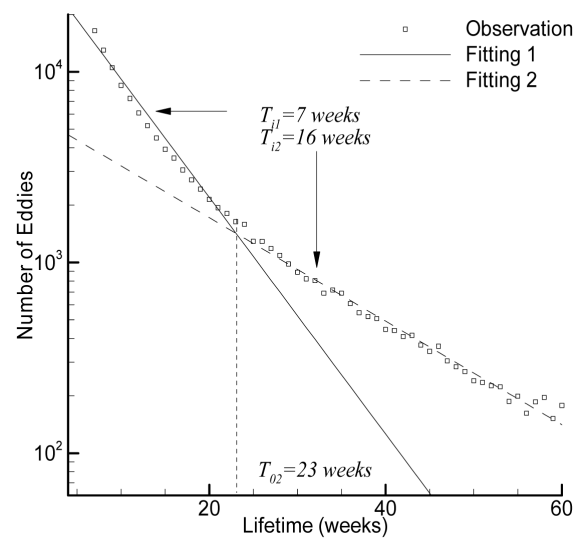

(c)

Figure 5. The same as in Figure 4 but for global oceans.

\subsection{Eddy Viscosity in the SCS}

Next, we use the data from Xiu et al. (2010) [27] to estimate eddy viscosity in the South China Sea (SCS). Some census statistics listed in their paper are useful for our study. A linear relationship between the eddy amplitude and eddy lifetime can be identified from this dataset. Using Equation (12) to fit the data give rise to an amplitude decaying rate of $a=0.08 \mathrm{~cm} /$ day $(a=0.09 \mathrm{~cm} /$ day $)$ for anticyclonic (cyclonic) eddies. Thus, $a=0.085 \mathrm{~cm} /$ day $=1.0 \times 10^{-8} \mathrm{~m} / \mathrm{s}$, on average, for eddies observed in the SCS (Table 2). The average amplitude and area for eddies in the SCS is approximately $15 \mathrm{~cm}$ and $127,880 \mathrm{~km}^{2}$, respectively [27]. We transformed the effective area of $127,880 \mathrm{~km}^{2}$ to the special area of $24,750 \mathrm{~km}^{2}$ (Table 2) with the regression relationship $r_{\mathrm{s}}=0.44 r_{\text {eff }}$ [4]. Substituting these average values in Equation (15), the eddy viscosity is estimated at approximately $343 \mathrm{~m}^{2} / \mathrm{s}$ in the SCS (Table 2), quite close to the average value for the two eddies discussed above.

As shown above, there is no significant difference for viscosity between the cyclonic and anticyclonic eddies. This is also similar to the results inferred from the data by Xiu et al. (2010) [27]; thus, eddy viscosity is insensitive to their polarization. 
Table 2. The average parameters of eddies in different regions.

\begin{tabular}{ccccccc}
\hline Dataset & $A_{a}(\mathrm{~cm})$ & $T_{a}(\mathrm{~d})$ & $\boldsymbol{a}(\mathrm{m} / \mathrm{s})$ & $S_{a}\left(\mathrm{~km}^{2}\right)$ & $\boldsymbol{r}_{\mathbf{a}}(\mathrm{km})$ & $\boldsymbol{V}_{\mathbf{a}}\left(\mathrm{m}^{2} / \mathrm{s}\right)$ \\
\hline ACC-C & 7.62 & 78.9 & $1.1 \times 10^{-8}$ & $3.2 \times 10^{3}$ & 32 & 100 \\
ACC-L & 16.7 & 73 & $2.6 \times 10^{-8}$ & $5.06 \times 10^{3}$ & 40 & 173 \\
GO-C & 6.76 & 83.6 & $0.9 \times 10^{-8}$ & $5.4 \times 10^{3}$ & 41.5 & 162 \\
GO-L & 16.7 & 76.6 & $2.5 \times 10^{-8}$ & $9.0 \times 10^{3}$ & 53.5 & 292 \\
SCS-X & 15 & 174 & $1.0 \times 10^{-8}$ & $24.8 \times 10^{3}$ & 87.4 & 343 \\
\hline
\end{tabular}

\subsection{Global Eddy Viscosities from the Li Dataset}

Next, we use the automatic identification and tracking data of the mesoscale eddies from the Li dataset to study the eddy viscosity. First, we use the data within the ACC region $\left(45^{\circ} \mathrm{S}-65^{\circ} \mathrm{S}\right)$. The eddy number vs amplitude distribution in the ACC region is plotted in Figure 2(a). It is obvious that the number of eddies and their amplitude obey an e-folding decay law, Equation (14a), where the intrinsic eddy amplitude is $A_{i}=8.7 \mathrm{~cm}$. Similarly, the eddy number and their area obey the e-folding decay law, Equation (14b), with the intrinsic eddy area $S_{i}=2.8 \times$ $10^{3} \mathrm{~km}^{2}$, Figure 2(b). In addition, the eddy numbers and lifetime obey the e-folding decay law, Equation (14c), with the intrinsic eddy lifetime $T_{i}=40$ days, Figure 2(c). From Equation (15a), the amplitude decaying rate $a=A_{i} / T_{i}=2.52 \times 10^{-8} \mathrm{~m} / \mathrm{s}$, and from Equation (15b) the length parameter $L=S_{i} / A_{i}=3.2 \times 10^{10} \mathrm{~m}$. Consequently, from Equation (15c) the eddy viscosity is approximately $64 \mathrm{~m}^{2} / \mathrm{s}$ in the ACC region.

Second, we apply this method to the global data. The census statistics are shown in Figure 3. Note that the larger the amplitude, the fewer the number of eddies. Although the eddy numbers vs amplitude obey the e-folding laws, there are critical values $\left(A_{02}=38 \mathrm{~cm}, S_{02}=36 \times 10^{3} \mathrm{~km}^{2}\right.$ and $T_{02}=78$ days) separating the e-folding decay laws into two segments. The intrinsic eddy amplitude is $A_{i l}=$ $9 \mathrm{~cm}$ and $A_{i 2}=16 \mathrm{~cm}$ for small amplitude and large amplitude eddies, these two segments join at amplitude of $38 \mathrm{~cm}$ (Figure 3(a)). Meanwhile, the eddy numbers vs area obey e-folding decay laws, where the intrinsic eddy area is $S_{i 1}=5.7 \times$ $10^{3} \mathrm{~km}^{2}$ and $S_{i 2}=23 \times 10^{3} \mathrm{~km}^{2}$ for small and large area eddies, respectively (Figure 3(b)). In addition, the eddy numbers vs lifetime obey e-folding decay laws (Figure 3(c)). The intrinsic eddy lifetime is $T_{i 1}=27$ days and $T_{i 2}=52$ days for small and large eddies, respectively. Thus, the eddy viscosity $v_{e}$ is $524 \mathrm{~m}^{2} / \mathrm{s}$ and $1099 \mathrm{~m}^{2} / \mathrm{s}$ for small and large eddies, respectively, in the global oceans.

\subsection{Global Eddy Viscosities from the Chelton Dataset}

The results discussed seem vary due to the difference in datasets created by different investigators. We also use the dataset calculated by Chelton et al. (2011) [4], note that the effective area should be transformed to the special area with the regression relationship $r_{s}=0.44 r_{\text {eff }}$ [4]. The results are shown in Figure 4. For eddies in the ACC region, the intrinsic amplitude, area and lifetime are $A_{i}=6.5$ 
$\mathrm{cm}, S_{i}=2.2 \times 10^{3} \mathrm{~km}^{2}$ and $T_{i}=8$ weeks, respectively. Thus, the eddy viscosity is approximately $97 \mathrm{~m}^{2} / \mathrm{s}$, which is relatively smaller than that obtained from Li's data in the ACC region (Table 3 ).

Similarly, the census statistics of the global mesoscale eddies are also calculated (Figure 5). The data and parameters are listed in Table 3. The eddy numbers vs lifetime obey e-folding decay laws with two segments (Figure 5(c)). The intrinsic eddy lifetime is $T_{i 1}=7$ weeks and $T_{i 2}=16$ weeks. The latter one is the same as the optimal value obtained by applying a stochastic model to the same dataset [34]. In contrast to the larger values of eddy viscosity obtained from the Li dataset, the eddy viscosities are only $184 \mathrm{~m}^{2} / \mathrm{s}$ and $270 \mathrm{~m}^{2} / \mathrm{s}$ (approximately $1 / 3$ and $1 / 4$ of the above corresponding values) for the small and large eddies for the global oceans from the Chelton's data.

It is concluded that viscosity calculated from different datasets is of the same order, although the difference is a bit large. Thus, using the intrinsic parameters rather than the census numbers is a better approach.

\subsection{Intrinsic Eddy Viscosity}

Lateral eddy viscosities derived in the discussion above are diverse in value, from $64 \mathrm{~m}^{2} / \mathrm{s}$ to $1099 \mathrm{~m}^{2} / \mathrm{s}$. Additionally, these lateral eddy viscosities are approximately one or two orders of magnitude smaller than the canonical value of approximately $10^{3}-10^{4} \mathrm{~m}^{2} / \mathrm{s}$ [7] [13]). One possible reason for this discrepancy is that eddy viscosity vary with the resolution. To illuminate this, we plotted eddy viscosity and the corresponding scales in Figure 6. It is obvious that all data points fit a simple power law $v_{e}=10^{-6} r_{s}^{1.8}$ for the eddy radius range of $\mathrm{O}\left(10^{-2}\right.$ $\left.10^{2} \mathrm{~km}\right)$.

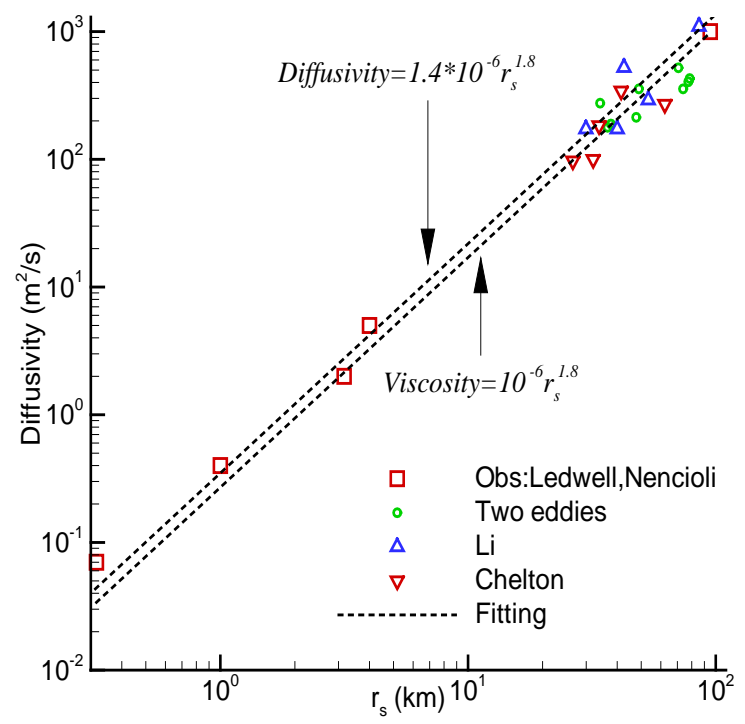

Figure 6. The eddy viscosity/diffusivity vs. scale, where the diffusivity from field observations are taken from Ledwell et al. (1998) [7] and Nencioli et al. (2013) [35], and the viscosity values are from present study. Both datasets obey the same power-law for the radius, but with different factors. 
Table 3. The intrinsic parameters of eddies in different regions.

\begin{tabular}{ccccccc}
\hline Dataset & $\mathrm{A}_{\mathrm{i}}(\mathrm{cm})$ & $\mathrm{S}_{\mathrm{i}}\left(\mathrm{km}^{2}\right)$ & $\mathrm{T}_{\mathrm{i}}(\mathrm{d})$ & $\mathrm{a}(\mathrm{m} / \mathrm{s})$ & $\mathrm{S}_{\mathrm{i}} / \mathrm{A}_{\mathrm{i}}(\mathrm{m})$ & $\mathrm{v}_{\mathrm{e}}\left(\mathrm{m}^{2} / \mathrm{s}\right)$ \\
\hline ACC-C & 6.5 & $2.2 \times 10^{3}$ & 56 & $1.34 \times 10^{-8}$ & $7.7 \times 10^{10}$ & 97 \\
ACC-L & 8.7 & $2.8 \times 10^{3}$ & 40 & $2.52 \times 10^{-8}$ & $3.2 \times 10^{10}$ & 173 \\
GO-C1 & 5 & $3.6 \times 10^{3}$ & 49 & $1.19 \times 10^{-8}$ & $1.9 \times 10^{11}$ & 184 \\
GO-C2 & 11.8 & $12 \times 10^{3}$ & 119 & $1.15 \times 10^{-8}$ & $2.7 \times 10^{11}$ & 254 \\
GO-L1 & 9 & $5.7 \times 10^{3}$ & 27 & $3.86 \times 10^{-8}$ & $6.3 \times 10^{10}$ & 524 \\
GO-L2 & 16 & $23 \times 10^{3}$ & 52 & $3.57 \times 10^{-8}$ & $1.4 \times 10^{11}$ & 1099 \\
\hline
\end{tabular}

This power law is also valid for the eddy diffusivities in the ocean. According to the observations [7], the lateral eddy diffusivity is $0.07 \mathrm{~m}^{2} / \mathrm{s}, 2 \mathrm{~m}^{2} / \mathrm{s}$, and $10^{3}$ $\mathrm{m}^{2} / \mathrm{s}$ at scales of 0.1 to $1 \mathrm{~km}, 1$ to $10 \mathrm{~km}$, and 30 to $300 \mathrm{~km}$, respectively. A recent study based on observations [35] indicated that lateral eddy diffusivity is $0.4 \mathrm{~m}^{2} / \mathrm{s}$ and $5 \mathrm{~m}^{2} / \mathrm{s}$ at scales of $1 \mathrm{~km}$ and $4 \mathrm{~km}$, respectively. If we take these scales as radius $r_{s}$, the diffusivities also obey a power-law of $k_{e}=1.4 \times 10^{-6} r_{s}^{1.8}$, as shown in Figure 4. On the other hand, if we take the scales as radius $r_{e f f}$ the diffusivities obey a power-law $k_{e}=7.2 \times 10^{-6} r_{s}^{1.8} \quad$ (figure not shown).

Both the eddy viscosity and diffusivity obey similar power laws, with slightly different constants in the front; in fact, viscosity is relatively smaller than the diffusivity. This can be understood in terms of the dissipation ratio $\Gamma$ and the ratio of buoyancy flux to turbulence production [36]; the ratio of viscosity to diffusivity is $(1-\Gamma)$, where $\Gamma$ is less than 0.2 for turbulence mixing [36] [37]. In the present case, according to Figure $6 \Gamma=0.28$ and 0.85 for $k_{e}=1.4 \times 10^{-6} r_{s}^{1.8}$ and $k_{e}=7.2 \times 10^{-6} r_{s}^{1.8}$, respectively; thus, mixing in the stratified ocean is weak, but it is slightly stronger than turbulence mixing in the homogeneous fluid in general.

As noted in the introduction, the diffusivities $k_{e}$ and $k_{O C}$ may be proportional to $L_{\text {eff }}^{n}(n<2)$. Our results show that $n=3 / 2$ for a $k_{m}$ of 10 to $10^{2} \mathrm{~m}^{2} / \mathrm{s}, n=5 / 3$ for a $k_{m}$ of $0.5-5 \mathrm{~m}^{2} / \mathrm{s}$, and $n=9 / 5$ for a $k_{m}$ of $10^{-2}$ to $10^{-1} \mathrm{~m}^{2} / \mathrm{s}$. It seems that when $k_{m}$ and the spatial resolution scale are sufficiently small, $n$ tends to be 2 , as we expected.

Additionally, we can extrapolate the above eddy mixing rates to small scales down to the molecule mixing rates, because it is well-known that the power laws are valid for a wide regime of $\mathrm{O}\left(10^{-2}-10^{2} \mathrm{~km}\right)$. Considering that the molecule diffusivity is $10^{-7} \mathrm{~m}^{2} / \mathrm{s}$ and the molecule viscosity is $10^{-6} \mathrm{~m}^{2} / \mathrm{s}$, it seems that power laws for eddy mixing could be used when the scale is larger than $0.3-1 \mathrm{~m}$. The extrapolation of the eddy mixing to molecule mixing implies that the above eddy mixing rates always hold until $r_{s}$ is smaller than $\mathrm{O}(1 \mathrm{~m})$. This extrapolation is physically sound, since that the turbulence is generally observed on scale larger than $\mathrm{O}(1 \mathrm{~m})$ in fluid dynamics. Thus, the above eddy mixing is expected to hold in the scales of $10^{-2}-10^{2} \mathrm{~km}$.

Finally, the intrinsic time has a relatively weaker relationship with the eddy 
scale. Because only time is directly proportional to length, the exponent $n<2$ holds according to Equation (15c). Both present regression lines imply a $1 / 5$ power law $T_{i} \propto r_{s}^{1 / 5}$ under the condition of $n=9 / 5$ for $k_{m}$. The larger scale eddies also have larger time scales.

\subsection{Climatological Distribution of Eddy Viscosity}

The eddy viscosity power law discussed above can be used to calculate the climatological distribution of eddy viscosity. First, we calculate the viscosity of each eddy on each time snapshot using the eddy parameters from Chelton and Li datasets. Then, each point of the eddy within the eddy perimeter (as indicated by effective radius $r_{\text {eff }}$ is recorded by the same viscosity. Finally, we calculate the climatological eddy viscosity by averaging the total viscosities within the whole time.

Figure 7 illuminates the climatological distribution of eddy viscosity for Chelton and Li datasets. In general, eddy viscosities are high at low latitudes, but low at high latitudes, except for the eddy rich systems (e.g. the Kuroshio and the Gulf Stream) where the eddy viscosities are relatively larger. The typical value is on order of $10^{2} \mathrm{~m}^{2} / \mathrm{s}$, consistent with the previously low values [9] [10]. It is found that the patterns are similar to these of mesoscale eddy diffusivities but about two order smaller [17] [19] [38]. If we use formula $k_{e}=7.2 \times 10^{-6} r_{s}^{1.8}$ to calculate eddy diffusivities, the results are ten times smaller than those obtained from the mixing length theory [17]. This again shows that eddy diffusivity is stronger than viscosity in oceanic mixing.

The present study shows a result of very low rate of viscosity than previous diffusivities [17] [19] [38]. However, from recent measurements on eddies, there is a clear testimony to the very low rate of viscous dissipation in the ocean [39].

\section{Discussion}

\subsection{Dependence of the Census}

In Section 3, we used the intrinsic eddy parameters to estimate the viscosity. We also used the averages of the eddy parameters to estimate the viscosity. To quantify the differences between these two approaches, we further compare the values obtained from these two different methods. According to the eddy census, the number of eddies obey the e-folding relations in Equation (14), the average parameters of eddies are

$$
\begin{aligned}
& A_{a}=\frac{1}{N} \int_{A_{0}}^{\infty} A N_{a}(A) \mathrm{d} A=A_{0}+A_{i} . \\
& S_{a}=\frac{1}{N} \int_{S_{0}}^{\infty} S N_{s}(S) \mathrm{d} S=S_{0}+S_{i} . \\
& T_{a}=\frac{1}{N} \int_{T_{0}}^{\infty} T N_{t}(T) \mathrm{d} T=T_{0}+T_{i} .
\end{aligned}
$$

where $N$ is the total eddy number. It is noted that the results depend on the initial and intrinsic parameters, but they are independent of the total eddy number. 


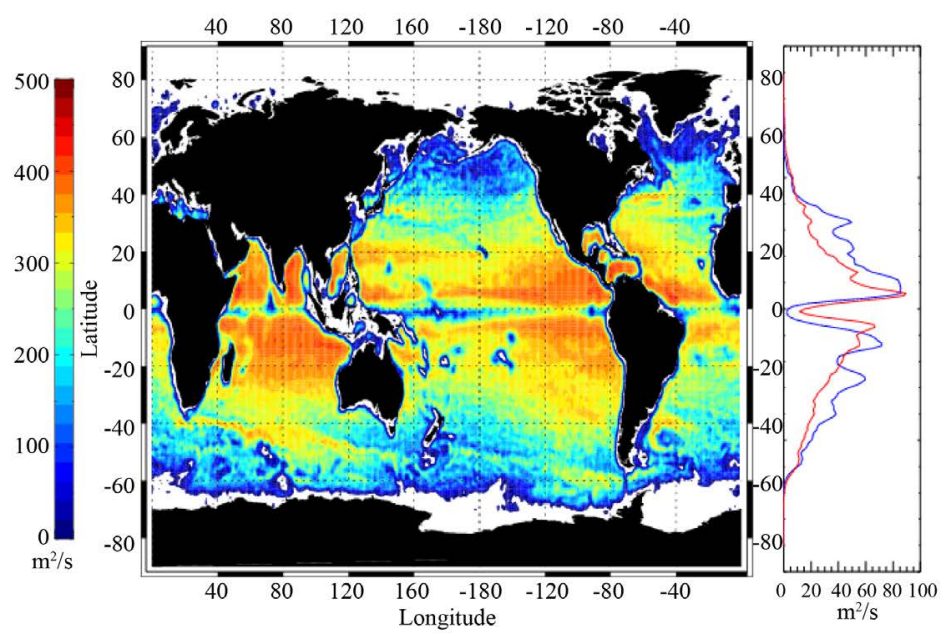

(a)

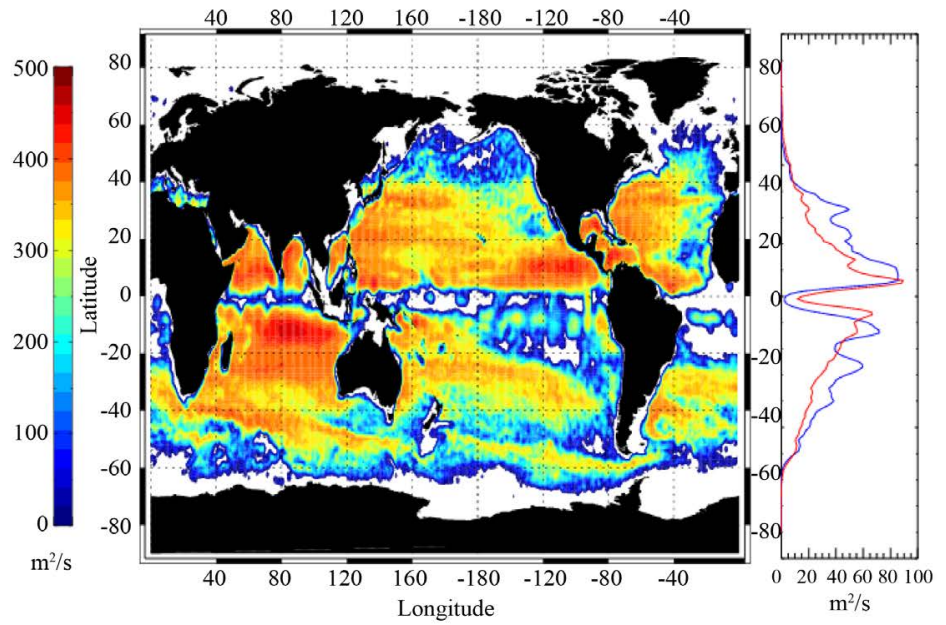

(b)

Figure 7. (a) Left panel: The eddy viscosity climatology for Chelton dataset. Right panel: The zonal average of eddy viscosity, red for Chelton dataset and blue for Li dataset; (b) The same as in (a) but for Li dataset.

The initial values are given by the identification criteria, as mentioned in the data subsection. From the census data (Tables 4-6) the averages agree quite well with the sums of the initial and intrinsic parameters, Equation (16), although initial values in the different datasets are quite different. For example, for lifetimes $>16$ weeks, the average lifetime is 32 weeks [4]. Thus, the intrinsic lifetime is 16 weeks according to Equation (16c), which is equal to the above fitting value in Figure 5(c); additionally, it is the same as the optimal value from a stochastic model with the same dataset [34]. Consequently, the results may not be sensitive to the eddy tracking dataset. The parameters are given by the averages as

$$
\begin{gathered}
a_{a}=A_{a} / T_{a}=\frac{A_{0}+A_{i}}{T_{0}+T_{i}}=\frac{1+A_{0} / A_{i}}{1+T_{0} / T_{i}} a . \\
L_{a}=S_{a} / A_{a}=\frac{S_{0}+S_{i}}{A_{0}+A_{i}}=\frac{1+S_{0} / S_{i}}{1+A_{0} / A_{i}} L .
\end{gathered}
$$


Table 4. Comparison of average and intrinsic amplitude of eddies.

\begin{tabular}{ccccc}
\hline Dataset & $A_{a}(\mathrm{~cm})$ & $A_{0}(\mathrm{~cm})$ & $A_{i}(\mathrm{~cm})$ & $\left(A_{0}+A_{i}\right) / A_{a}$ \\
\hline ACC-C & 7.62 & 1 & 6.5 & 1.0 \\
ACC-L & 16.7 & 6 & 8.7 & 0.9 \\
\hline
\end{tabular}

Table 5. Comparison of average and intrinsic area of eddies.

\begin{tabular}{ccccc}
\hline Dataset & $S_{a}\left(\mathrm{~km}^{2}\right)$ & $S_{0}\left(\mathrm{~km}^{2}\right)$ & $S_{i}\left(\mathrm{~km}^{2}\right)$ & $\left(S_{0}+S_{i}\right) / S_{a}$ \\
\hline ACC-C & $3.2 \times 10^{3}$ & $1.1 \times 10^{3}$ & $2.2 \times 10^{3}$ & 1.0 \\
ACC-L & $5.06 \times 10^{3}$ & $2.2 \times 10^{3}$ & $2.8 \times 10^{3}$ & 1.0 \\
\hline
\end{tabular}

Table 6. Comparison of average and intrinsic lifetime of eddies.

\begin{tabular}{ccccc}
\hline Dataset & $T_{\mathrm{a}}$ (days) & $T_{0}$ (days) & $T_{\mathrm{i}}$ (days) & $\left(T_{0}+T_{\mathrm{i}}\right) / T_{a}$ \\
\hline ACC-C & 79 & 28 & 56 & 1.1 \\
ACC-L & 73 & 30 & 40 & 1.0 \\
\hline
\end{tabular}

Table 7. The relative parameters in different dataset.

\begin{tabular}{cccc}
\hline Dataset & $A_{02} / A_{\text {in }}$ & $S_{02} / S_{12}$ & $T_{02} / T_{12}$ \\
\hline GO-C2 & 1.86 & 1.53 & 1.44 \\
GO-L2 & 2.38 & 1.56 & 1.50 \\
\hline
\end{tabular}

$$
v_{a}=\frac{-C}{4 \pi} L_{a} a_{a}=\frac{1+S_{0} / S_{i}}{1+T_{0} / T_{i}} v_{e} .
$$

All of averages will be dependent on the intrinsic parameters, but independent of the artificial parameters $A_{0}, T_{0}$ and $S_{0}$, under the condition of

$$
A_{0} / A_{i} \approx S_{0} / S_{i} \approx T_{0} / T_{i} \text {. }
$$

or,

$$
A_{0} / A_{i} \ll 1, S_{0} / S_{i} \ll 1, T_{0} / T_{i} \ll 1
$$

For example (Table 7), the Li data has $S_{02} / S_{i}=1.57$ (Figure 3(b)) and $T_{02} / T_{i}=$ 1.5 (Figure 3(c)) and the Chelton data has $S_{02} / S_{i}=1.53$ (Figure 5(b)) and $T_{02} / T_{i}=$ 1.44 (Figure 5(c)); in both cases, the values are consistent with the condition in Equation (18a). Thus, we may use the average parameters of the eddy properties as the corresponding intrinsic parameters under the conditions of Equation (18).

\subsection{Dependence of Datasets}

In this study, different eddy datasets are used, and these datasets are derived from different identification and tracking algorithms. Moreover, the critical values used to identify the coherent structures as eddies are quite different in the various datasets. For this technical reason, the coherent structures associated with smaller amplitudes and sizes are not identified as eddies in the Li dataset, but they are identified as eddies in the Chelton data. Consequently, eddies in the 
Li dataset have larger amplitudes and sizes and relatively shorter lifetimes compared to those in the Chelton dataset (Tables 4-6).

As we can see from Equation (15), the larger the eddy size, the larger the viscosity is. This is the reason why the viscosities are larger in the Li dataset. Additionally, the larger eddies (e.g., the eddies in regime 2) also experience larger viscosities in the same datasets, as shown in Table 2 and Table 3.

Both datasets show good consistency between the average and intrinsic parameters in Equation (16). The intrinsic parameters are independent of the critical values of identification, which are artificially chosen in the different datasets. It was expected that only the intrinsic parameters could be universal and independent of the mesoscale eddy datasets. The mixing rates, contrary to our expectations, are also universal and independent of the datasets.

\subsection{Impacts of Other Dynamics}

Our method of estimating eddy viscosity from data is based on an implicit assumption that the dissipation of an eddy's total energy is due to the viscosity only, and there is no net energy supplied from other mechanisms, such as wind forcing, energy genesis from the baroclinic instability of the flow and merging of ambient eddies, or the energy lost due to bottom friction, and the eddy splitting over time.

However, it is apparent from the two eddy examples shown above that eddy evolutions are not always characterized by monotonic decay with time. The amplitude of the eddy might increase from time to time (Figure 1). Thus, the use of the amplitude-lifetime relations might lead to an underestimation of the amplitude decaying rate $a$, which is not the case for the values of decaying rate a from this case study. For example, $a=0.009 \mathrm{~cm} /$ day as inferred from a long-lived eddy (Souza et al., 2011 [30]), which represents only approximately $1 / 40$ to $1 / 20$ of those reported in the present study. Thus, eddy viscosity reported in this study might serve as a lower bound of the true eddy viscosity in the ocean. For instance, the viscosity in ACC is approximately $173 \mathrm{~m}^{2} / s$ as inferred from the $\mathrm{Li}$ dataset, which is exactly the lowest value obtained from the example eddies in Figure 1.

The eddy viscosity in Equation (10) depends also on ratio $C$ of total mechanical energy to EKE. To precisely estimate eddy viscosity, we need to use individual $C$ for each eddy. In this study, we also use a constant $C=2.7$, which is from a global average with 2-layer ocean model [40]. According to the estimation (Figure 4(c) in [40]), $C$ is approximately linear increase with latitude from 1.5 to 4 . So the above result might be varied with a factor of 2 .

\section{Conclusion}

We test the hypothesis that eddy viscosity is proportional to $r_{s}^{n}(n<2)$ using eddy datasets. The dimensional eddy viscosities in different oceans obey the power law of $v_{e}=10^{-6} r_{s}^{1.8}$, which agrees well with the power law of observed diffusivities of $k_{e}=1.4 \times 10^{-6} r_{s}^{1.8}$ or $k_{e}=7.2 \times 10^{-6} r_{s}^{1.8}$. Additionally, the extra- 
polation of the eddy mixing to molecule mixing implies that the above eddy mixing rates always hold until the value of $r_{s}$ is less than $\mathrm{O}(1 \mathrm{~m})$. Since such parameterization is valid from very small scale to very large scale, the mixing rates with the new parameterizations are suggested to use in numerical schemes. It is expected that the new parameterization may improve the numerical simulations accordingly. Compared with the larger value $\left(10^{3}-10^{4} \mathrm{~m}^{2} / \mathrm{s}\right)$ of eddy viscosity commonly used in coarse and eddy permitting resolution models, lateral eddy viscosity inferred from satellite observations in the open ocean is on the order of $10^{2}-10^{3} \mathrm{~m}^{2} / \mathrm{s}$. It implies that oceanic eddy mixing is more like strong diffusion than ordinary turbulence. The census of the mesoscale eddies shows, in general, that the eddy numbers obey e-folding decay laws in terms of their amplitude, area and lifetime, regardless of the regions and the choice of datasets. The present results are useful for the parameterizations in the numerical ocean models with horizontally variable resolutions.

\section{Acknowledgements}

We thank AVISO for providing the SLA data (http://www.aviso.oceanobs.com/). We thank Prof. R. X. Huang at WHOI for useful comments. This work was supported by the National Foundation of Natural Science (No. 41376017).

\section{References}

[1] Chelton, D.B., Schlax, M.G., Samelson, R.M. and de Szoeke, R.A. (2007) Global Observations of Large Oceanic Eddies. Geophysical Research Letters, 34, 87-101.

[2] Pujol, M.I., Faugère, Y., Taburet, G., Dupuy, S., Pelloquin, C., Ablain, M., et al. (2016) DUACS DT2014: The New Multi-Mission Altimeter Dataset Reprocessed over 20 Years. Ocean Science, 12, 1067-1090. https://doi.org/10.5194/os-12-1067-2016

[3] Roemmich, D. and Gilson, J. (2001) Eddy Transport of Heat and Thermocline Waters in the North Pacific: A Key to Interannual/Decadal Climate Variability? Journal of Physical Oceanography, 31, 675-687. https://doi.org/10.1175/1520-0485(2001)031<0675:ETOHAT>2.0.CO;2

[4] Chelton, D.B., Schlax, M.G. and Samelson, R.M. (2011) Global Observations of Nonlinear Mesoscale Eddies. Progress in Oceanography, 91, 167-216. https://doi.org/10.1016/j.pocean.2011.01.002

[5] Hallberg, R. and Gnanadesikan, A. (2006) The Role of Eddies in Determining the Structure and Response of the Wind-Driven Southern Hemisphere Overturning: Results from the Modeling Eddies in the Southern Ocean (MESO) Project. Journal of Physical Oceanography, 36, 2232-2252. https://doi.org/10.1175/JPO2980.1

[6] Treguier, A.M., Boning, C.W., Bryan, F., Danabasoglu, G., Drange, H. and Taguchi, B. (2014) CLIVAR WGOMD Workshop on High Resolution Ocean Climate Modelling: Outcomes and Recommendations. CLIVAR Exchanges, 19, 3-6.

[7] Ledwell, J.R., Watson, A.J. and Law, C.S. (1998) Mixing of a Tracer in the Pycnocline. Journal of Geophysical Research: Oceans, 103, 21499-21529.

[8] Brown, E.D. and Owens, W.B. (1981) Observations of the Horizontal Interactions between the Internal Wave Field and the Mesoscale Flow. Journal of Physical Oceanography, 11, 1474-1480. 
https://doi.org/10.1175/1520-0485(1981)011<1474:OOTHIB >2.0.CO;2

[9] Ferrari, R. and Polzin, K.L. (2005) Finescale Structure of the T-S Relation in the Eastern North Atlantic. Journal of Physical Oceanography, 35, 1437-1454. https://doi.org/10.1175/JPO2763.1

[10] Visbeck, M., Marshall, J., Haine, T. and Spall, M. (1997) Specification of Eddy Transfer Coefficients in Coarse-Resolution Ocean Circulation Models. Journal of Physical Oceanography, 27, 381-402.



[11] Bryan, K. (1987) Potential Vorticity in Models of the Ocean Circulation. Quarterly Journal of the Royal Meteorological Society, 113, 713-734.

[12] Zhurbas, V. and Oh, I.S. (2004) Drifter-Derived Maps of Lateral Diffusivity in the Pacific and Atlantic Oceans in Relation to Surface Circulation Patterns. Journal of Geophysical Research: Oceans, 109, C05015.

[13] Cole, S.T., Wortham, C., Kunze, E. and Owens, W.B. (2015) Eddy Stirring and Horizontal Diffusivity from Argo Float Observations: Geographic and Depth Variability. Geophysical Research Letters, 42, 3989-3997.

[14] Redi, M.H. (1982) Oceanic Isopycnal Mixing by Coordinate Rotation. Journal of Physical Oceanography, 12, 1154-1158. https://doi.org/10.1175/1520-0485(1982)012<1154:OIMBCR>2.0.CO;2

[15] Nakamura, N. (1996) Two-Dimensional Mixing, Edge Formation, and Permeability Diagnosed in an Area Coordinate. Journal of the Atmospheric Sciences, 53, 1524-1537. https://doi.org/10.1175/1520-0469(1996)053<1524:TDMEFA>2.0.CO;2

[16] Osborn, T.R. and Cox, C.S. (1972) Oceanic Fine Structure. Geophysical \& Astrophysical Fluid Dynamics, 3, 321-345. https://doi.org/10.1080/03091927208236085

[17] Bates, M., Tulloch, R., Marshall, J. and Ferrari, R. (2014) Rationalizing the Spatial Distribution of Mesoscale Eddy Diffusivity in Terms of Mixing Length Theory. Journal of Physical Oceanography, 44, 1523-1540. https://doi.org/10.1175/JPO-D-13-0130.1

[18] Marshall, J., Shuckburgh, E., Jones, H. and Hill, C. (2006) Estimates and Implications of Surface Eddy Diffusivity in the Southern Ocean Derived from Tracer Transport. Journal of Physical Oceanography, 36, 1806-1821. https://doi.org/10.1175/JPO2949.1

[19] Abernathey, R.P. and Marshall, J. (2013) Global Surface Eddy Diffusivities Derived from Satellite Altimetry. Journal of Geophysical Research: Oceans, 118, 901-916.

[20] Shuckburgh, E. and Haynes, P. (2003) Diagnosing Transport and Mixing using a Tracer-Based Coordinate System. Physics of Fluids, 15, 3342-3357. https://doi.org/10.1063/1.1610471

[21] Fox-Kemper, B., Backman, S.D., Pearson, B. and Reckinger, S. (2014) Principles and Advances in Subgrid Modelling for Eddy-Rich Simulations. Clivar Exchanges, 19, 42-46.

[22] Li, Q.Y., Sun, L., Liu, S.S., Xian, T. and Yan, Y.F. (2014) A New Mononuclear Eddy Identification Method with Simple Splitting Strategies. Remote Sensing Letters, 5, 65-72. https://doi.org/10.1080/2150704X.2013.872814

[23] Li, Q.Y. and Sun, L. (2015) Watershed Strategy for Oceanic Mesoscale Eddy Splitting. Ocean Science, 11, 269-273. https://doi.org/10.5194/os-11-269-2015

[24] Li, Q.-Y., Sun, L. and Lin, S.-F. (2016) GEM: A Dynamic Tracking Model for Mesoscale Eddies in the Ocean. Ocean Science, 12, 1249-1267.

https://doi.org/10.5194/os-12-1249-2016 
[25] Ponte, R.M., Wunsch, C. and Stammer, D. (2007) Spatial Mapping of Time-Variable Errors in Jason-1 and TOPEX/Poseidon Sea Surface Height Measurements. Journal of Atmospheric and Oceanic Technology, 24, 1078-1085. https://doi.org/10.1175/JTECH2029.1

[26] Chaigneau, A., Gizolme, A. and Grados, C. (2008) Mesoscale Eddies off Peru in Altimeter Records: Identification Algorithms and Eddy Spatio-Temporal Patterns. Progress in Oceanography, 79, 106-119. https://doi.org/10.1016/j.pocean.2008.10.013

[27] Xiu, P., Chai, F., Shi, L., Xue, H. and Chao, Y. (2010) A Census of Eddy Activities in the South China Sea during 1993-2007. Journal of Geophysical Research: Oceans, 115, C03012.

[28] Zhang, Z., Zhang, Y., Wang, W. and Huang, R.X. (2013) Universal Structure of Mesoscale Eddies in the Ocean. Geophysical Research Letters, 40, 3677-3681.

[29] Wang, Z., Li, Q., Sun, L., Li, S., Yang, Y. and Liu, S. (2015) The Most Typical Shape of Oceanic Mesoscale Eddies from Global Satellite Sea Level Observations. Frontiers of Earth Science, 9, 202-208. https://doi.org/10.1007/s11707-014-0478-Z

[30] Sun, L. (2011) A Typhoon-Like Vortex Solution of Incompressible 3D Inviscid Flow. Theoretical and Applied Mechanics Letters, 1, Article ID: 042003. https://doi.org/10.1063/2.1104203

[31] Wu, J.Z., Ma, H.Y. and Zhou, M.D. (2007) Vorticity and Vortex Dynamics. Springer Science \& Business Media, Berlin Heidelberg, Vol. 14, 776 p.

[32] Xu, C., Shang, X.D. and Huang, R.X. (2014) Horizontal Eddy Energy Flux in the World Oceans Diagnosed from Altimetry Data. Scientific Reports, 4, 5316. https://doi.org/10.1038/srep05316

[33] Souza, J.M.A.C.D., De Boyer Montegut, C. and Le Traon, P.Y. (2011) Comparison between Three Implementations of Automatic Identification Algorithms for the Quantification and Characterization of Mesoscale Eddies in the South Atlantic Ocean. Ocean Science, 7, 317-334. https://doi.org/10.5194/os-7-317-2011

[34] Samelson, R.M., Schlax, M.G. and Chelton, D.B. (2014) Randomness, Symmetry, and Scaling of Mesoscale Eddy Life Cycles. Journal of Physical Oceanography, 44, 1012-1029. https://doi.org/10.1175/JPO-D-13-0161.1

[35] Nencioli, F., d'Ovidio, F., Doglioli, A.M. and Petrenko, A.A. (2013) In Situ Estimates of Submesoscale Horizontal Eddy Diffusivity across an Ocean Front. Journal of Geophysical Research: Oceans, 118, 7066-7080.

[36] Osborn, T.R. (1980) Estimates of the Local Rate of Vertical Diffusion from Dissipation Measurements. Journal of Physical Oceanography, 10, 83-89. https://doi.org/10.1175/1520-0485(1980)010<0083:EOTLRO >2.0.CO;2

[37] Sun, L. (2014) Identify the Diapycnical Eddy Diffusivities by Salt Fingers and Turbulence with Vertical Microstructure Measurements.

[38] Klocker, A. and Abernathey, R. (2014) Global Patterns of Mesoscale Eddy Properties and Diffusivities. Journal of Physical Oceanography, 44, 1030-1046. https://doi.org/10.1175/JPO-D-13-0159.1

[39] Rossby, T. (2016) Visualizing and Quantifying Oceanic Motion. Annual Review of Marine Science, 8, 35-57. https://doi.org/10.1146/annurev-marine-122414-033849

[40] Xu, C., Shang, X.D. and Huang, R.X. (2011) Estimate of Eddy Energy Generation/Dissipation Rate in the World Ocean from Altimetry Data. Ocean Dynamics, 61, 525-541. https://doi.org/10.1007/s10236-011-0377-8 